\title{
A high-throughput method for fingerprinting of rhizobial free fatty acids by the combination of a thin film chemical deposition technique and matrix-assisted laser desorption/ionization time-of flight mass spectrometry
}

\author{
Aleksey Gladchuk ${ }^{1 \S}$, Julia Shumilina ${ }^{2,3 \S}$, Alena Kusnetsova ${ }^{2 \S}$, Ksenia Bureiko ${ }^{2,4}$, Alexander \\ Tsarev,3, ,Irina Alexandrova1, Larisa Leonova ${ }^{2}$, Vladimir A. Zhukov5, Igor A. Tikhonovich,6, \\ Claudia Birkemeyer ${ }^{7}$ Ekaterina Podolskaya ${ }^{1,8}$ and Andrej Frolov ${ }^{2,3^{*}}$ \\ 1 Institute of Toxicology, Federal Medical-Biological Agency of Russia; ek.podolskaya@gmail.com, \\ aleglad24@gmail.com, analekt@mail.ru \\ 2 Department of Biochemistry, St. Petersburg State University; schumilina.u@yandex.ru, alena_kyy@mail.ru, \\ ksenya.bu@gmail.com, alexandretsarev@gmail.com, larisa_leonova@mail.ru, a.a.frolov@spbu.ru, \\ 3 Department of Bioorganic Chemistry, Leibniz Institute of Plant Biochemistry; afrolov@ipb-halle.de \\ 4 Department of Invertebrate Zoology, St. Petersburg State University; ksenya.bu@gmail.com \\ 5 All-Russia Research Institute for Agricultural Microbiology; vladimir.zhukoff@gmail.com, \\ arriam2008@yandex.ru \\ 6 Department of Genetics and Biotechnology, St. Petersburg State University; arriam2008@yandex.ru \\ 7 Institute of Analytical Chemistry, Faculty of Chemistry and Mineralogy, Universität Leipzig; \\ birkemeyer@chemie.uni-leipzig.de \\ 8 Institute of Analytical Instrumentation, Russian Academy of Sciences; ek.podolskaya@gmail.com \\ * Correspondence: afrolov@ipb-halle.de and a.a.frolov@spbu.ru; Tel.: +49-3455-582-1350 \\ $\S$ These authors contributed equally to the manuscript
}

\begin{abstract}
Fatty acids (FAs) represent an important class of metabolites, impacting on membrane building blocks and cellular regulatory networks. In nature, prokaryotes are characterized with the most impressing FA structural diversity and the highest relative contents of free fatty acids (FFAs). Thereby, nitrogen-fixing bacteria (order Rhizobiales), often found in symbiosis with legumes, attract a special interest. Indeed, FAs impact on the structure of rhizobial nodulation factors, required for successful infection of plant root. Although the FA patterns can be addressed by GCand LC-MS, these methods are time-consuming and suffer from compromised sensitivity, low stability of derivatives and artifacts. In contrast, MALDI-TOF-MS represents an excellent platform for high-efficient metabolite fingerprinting, also applicable to FFAs. Therefore, here we propose a simple and straightforward protocol for high-throughput relative quantification of FFAs in rhizobia by the combination of Langmuir technology and MALDI-TOF-MS, which is featured with high sensitivity, accuracy and precision of quantification. Here we propose a step by step procedure comprising rhizobia culturing, pre-cleaning, extraction, sample preparation, mass spectrometric analysis, data processing and post-processing. To demonstrate the analytical potential of the protocol we illustrate it by a case study - comparison the FFA metabolomes of two rhizobia species - Rhizobium leguminosarum and Sinorhizobium meliloti.
\end{abstract}

Keywords: bacteria; barium monocarboxylates; chemical deposition techniques; free fatty acids (FFAs); Langmuir film technology; matrix-assisted laser desorption/ionization-time of flight mass spectrometry (MALDI-TOF-MS); metabolic fingerprinting; rhizobia 


\section{Introduction}

Fatty acids (FAs) represent one of the most important metabolite classes in living organisms [1]. Indeed, on one hand, these compounds are readily involved in a wide array of enzymatic reactions yielding esters of aliphatic, cyclic and aromatic alcohols, critically impacting on the structure of membrane and reserve lipids [2]. On the other, in non-esterified form, FAs serve as precursors of hormones and modulators- key players of plant [3] and animal [4] regulatory pathways, involved in all vital physiological reactions. Not less important is the role of free fatty acids (FFAs) as low molecular weight effectors, directly involved in immunity responses in all kingdoms of living organisms [4]. The cellular pool of fatty acids can be relatively easily accessed either directly (FFAs) of after degradation of esters (bound, esterified FA fraction) [5]. Therefore, FFAs attract a special attention of biologists and analysts [6].

Due to a high structural heterogeneity of the ester-bound fraction and essential contribution of FFAs in the overall FA patterns [7], prokaryotes comprise a remarkable group of organisms in respect of the FA composition. Bacterial lipids are represented with phospholipids, their aminoacylated derivatives, glycerolipids, betaine lipid diacylglyceryl- $N, N, N$-trimethylhomoserine (DGTS), as well as ornithine lipids, lipopolysaccharides, sphingolipids, sulfonolipids and phenolic lipids [8]. The patterns of bacterial FFAs are strongly dominated with higher linear saturated (C12-C28) and unsaturated (C14-C28), branched (mostly saturated, containing up to 52 carbon atoms), hydroxylated and cyclic acids [7]. In the context of FA patterns, rhizobial bacteria represent one of the most interesting groups of prokaryotes (Figure 1). These microorganisms readily form symbiotic associations with roots of higher plants, which are most often represented by legume species [9]. Formation of root nodules, i.e. morphological structures, characteristic for legume rhizobial symbiosis, is a complex process, at the early steps governed by so-called nodulation (Nod) factors - sulfated chitooligosaccharides which are, for example, in the case of Rhizobium meliloti, mono-N-acylated by unsaturated C16 or by a series of C18 to C26 (ळ-1)-hydroxylated fatty acids [10]. Therefore, analysis of FA composition in rhizobial cells reminds an important task of analytical science.

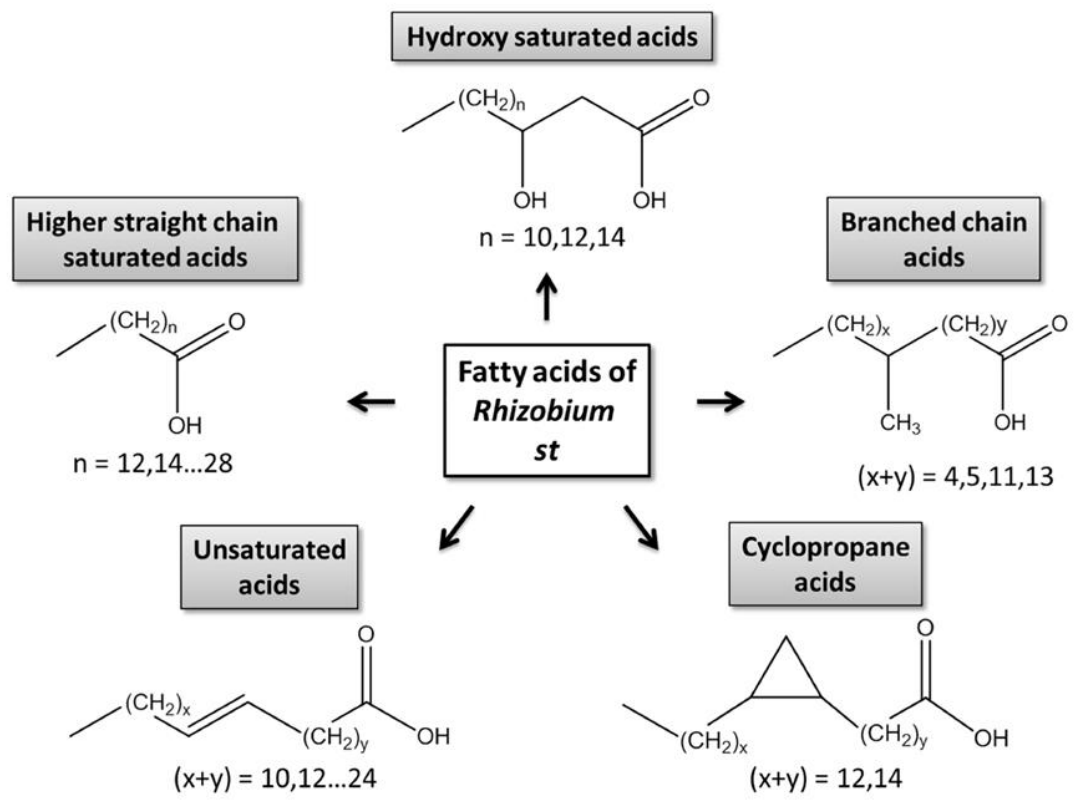

Figure 1. Structural class of bacterial fatty acids

In modern bioanalytical chemistry, analysis of FFA composition most often relies on gas chromatography - mass spectrometry (GC-MS) [11]. Thereby, short-chained volatile acids can be analyzed by head space techniques [12], whereas the long-chained ones can be assessed by liquid 
injection after appropriate derivatization [13]. The latter approach, relying on detection of methyl esters, proved to be efficient in analysis of branched fatty acids in bacterial membranes [14]. However, implementation of derivatization procedures in experimental workflows dramatically reduces sample throughput and might trigger transesterification of lipids, as well as isomerization and oxidation of unsaturated FFAs [15, 16]. Although hydrophilic interaction liquid chromatography (HILIC) [17] and reversed phase high-performance liquid chromatography (RP-HPLC) [18], coupled on-line to electrospray ionization mass spectrometry (ESI-MS) or tandem MS (MS/MS) in a multiple reaction monitoring (MRM) mode [19] can be also employed in quantification of FFAs, these techniques lack sensitivity or fatty acid metabolome coverage in comparison to traditional GC-MS-based workflows [20].

These bottle necks of GC-MS- and LC-MS-based approaches for FA analysis can be efficiently overcome by implementation of matrix-assisted laser desorption/ionization time-of flight mass spectrometry (MALDI-TOF-MS) in combination with Langmuir-Blodgett technology [21]. Recently, we have shown that Langmuir-Blodgett films can be not only efficiently implemented in selective enrichment of phosphopeptides [22], protein adducts of organophosphorous compounds [23] and chlorinated insecticides [24], but also proved to be an efficient tool for high-throughput and sensitive fingerprinting of free fatty acids as their barium monocarboxylates in positive ion mode [25]. Therefore, here we extend this approach to analysis of FFA composition of rhizobial bacteria and propose a comprehensive protocol for fingerprinting of FFAs in cultured cells by MALDI-TOF-MS.

\section{Experimental Design}

Langmuir technology in combination with MALDI-TOF-MS is the core methodology, underlying the presented here protocol [25]. In general, it gives access to unique structures, characterized with a high regularity at the molecular level [26]. This can be exemplified by formation of monolayers, accompanying the reaction of stearic acid with a trivalent metal salt (e.g. lanthanium trinitrate) at the interface of organic and aqueous phases [23]. Such monolayers are well-accessible under standard laboratory conditions. The preparation protocol typically assumes an overlay of aqueous salt buffer with FA in hexane or other organic solvent [27]. Under these conditions, hydrophilic carboxylic group of the acid is involved in hydrogen bonding with water and builds ion pairs with dissolved divalent cations, whereas the nonpolar aliphatic chain interacts with the organic phase. Formation of these ion pairs is fast, quantitative and for long-chained FAs (C12 and higher) yields non-soluble in water (but soluble in organic solvents) monolayers. Importantly, only free fatty acids are involved in this reaction whereas the most of other metabolites are not incorporated in the monolayers. After collapsing, such monolayers can be collected in polypropylene tubes, reconstituted and analyzed by MALDI-TOF-MS.

Although the soft reaction conditions make Langmuir technology a promising tool in sample preparation for mass spectrometric analysis, its implementation in analytical practice required, however, principal changes in the monolayer preparation strategy. First, as we have shown in our recent work [21], the cationic component needs to be replaced by divalent metal, ideally barium. Indeed, these cations form mostly monocarboxylates and no mixed salts, i.e. yielding efficiently ionizable monolayers. Such monocarboxylate monolayers can be desorbed from the surface of conventional MALDI target by UV laser, and can be, therefore, analyzed by MALDI-TOF-MS instrumentation. The resulting spectra are well-interpretable and can be used for quantitative estimations. 
Unfortunately, short-chain and unsaturated FAs don't form solid monolayers. Therefore, their salts remain on the surface of the aqueous phase, when such monolayers are collapsed. This and several other limitations can be overcome by transfer of the Langmuir technology from the planar setup (e.g. in a Petri dish) to a droplet format [25]. This allows formation of monolayers directly on a MALDI target without any losses of the sample. Secondly, due to a convex surface of the droplet, higher concentrations of the metal ions at the interphase can be achieved. Thereby, sample consumption is rather low (not more than $1 \mu \mathrm{L}$ per sample). Finally, the structure of the monolayers remains regular, that results in high reproducibility of analysis.

The overall experimental setup behind the proposed protocol includes several principal steps (Figure 2): culturing of rhizobia on agar and in aqueous nutritional medium, pre-cleaning of bacterial cells, hexane extraction from bacterial pellet of aqueous suspension, application of the sample to the MALDI target, mass spectrometric analysis, qualitative and quantitative analysis and statistical interpretation (post-processing).

\subsection{Materials}

- $\quad$ Safe-lock $2 \mathrm{~mL}$ polypropylene tubes (Eppendorf, obtained from Helicon, Moscow, Russia; Cat. No.: Epp 0030 120.094)

- $\quad$ Cell Culture Dishes, 90x14 mm (Noex Pharma, Moscow, Russia)

- $\mathrm{n}$-Hexane (HPLC grade, $\geq 95 \%$ purity, Merck, obtained from ChimMed, St. Petersburg, Russia; Cat. No.: 270504)

- Dipotassium hydrogen phosphate anhydrous $(\geq 98 \%$, ACS grade, VWR International, obtained from Helicon, Moscow, Russia; Cat. No.: Am-O705-0.5)

- Agar for bacteriology (VWR International, obtained from Helicon, Moscow, Russia; Cat. No.: Am-J637-0.5)

- Magnesium sulfate anhydrous (reagent grade, $\geq 97 \%$, Merck, obtained from ChimMed, St. Petersburg, Russia; Cat. No.: 208094)

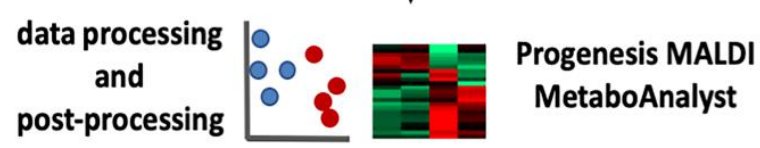

Figure 2.Experimental setup, applied for analysis of rhizobial free fatty acids by the combination of Langmuir technology and MALDI-TOF-MS.

- Calcium carbonate (ACS reagent grade, $\geq 99.0 \%$, Merck, obtained from ChimMed, St. Petersburg, Russia; Cat. No.: 239216)

- $\quad$ Sodium chloride (ACS reagent grade, $\geq$ 99.0\%, Merck, obtained from ChimMed, St. 
Petersburg, Russia; Cat. No.: S9888)

- D-mannitol ( $\geq 98 \%$, Merck, obtained from ChimMed, St. Petersburg, Russia; Cat. No.: M4125)

- Yeast extract (Biospringer, acquired from Helicon, Russia; Cat. No.: H-0601MG-0.5)

- Barium acetate (Merck, obtained from ChimMed, St. Petersburg, Russia; Cat. No.: 243671)

- Parafilm M (Bemis Company, obtainedfrom Helicon, Moscow, Russia; Cat. No.: PM996)

- 2,5-dihydroxybenzoic acid (mass spectrometry grade, Bruker Daltonik GmbH, Bremen, Germany; Cat. No.: 8201346)

- Acetonitrile (LC-MS grade, Merck, obtained from ChimMed, St. Petersburg, Russia; Cat. No.: 100029)

- Bacterial strains Rhizobium leguminosarum bv. viciae RCAM1026 and Sinorhizobium meliloti RCAM1021 (the collection of the All-Russian Research Institute for Agricultural Microbiology, St. Petersburg, Russia). Keep bacteria at $4^{0} \mathrm{C}$ in Petri dishes on agar nutrient medium).

\subsection{Equipment}

- Vortex mixer Vortex-genie 2 (Scientific Industries, acquired through ThermoFisher Scientific, Schwerte, Germany)

- Ultrasound bath Sonorex TK-30 (BANDELIN electronic GmbH \& Co. KG, Berlin, Germany)

- Benchtop incubator shaker Innova 40 (Eppendorf,obtained from Helicon, Moscow, Russia)

- Tabletop centrifuge (5415 R, Eppendorf,obtained from Helicon, Moscow, Russia)

- MTP 384 polished steel BC target (Bruker Daltonik GmbH, Bremen, Germany)

- MALDI TOF/TOF mass spectrometer UltrafleXtreme (Bruker Daltonik GmbH, Bremen, Germany).

- Microplate spectrophotometer PowerWave HT (BioTek Instruments, obtained from Bioline LLC, St.Petersburg, Russia)

\subsection{Softwares}

- FlexControl 3.4 and FlexAnalysis 3.4 (Bruker Daltonik GmbH, Bremen, Germany)

- Progenesis MALDI v1.2 (Nonlinear Dynamics, Newcastle upon Tyne, UK)

- MetaboAnalyst 4.0 (https://www.metaboanalyst.ca/MetaboAnalyst/home.xhtml)

\subsection{Costs}

Overall price of consumables for analysis of one sample can be estimated as $2 €$. These costs include reagents and plastic (tubes, pipette tips).

\section{Procedure}

\subsection{Sterilize (180 $\mathrm{min})$}

Sterilize nutritional medium \# 79 , tips and tubes by autoclaving at $121^{\circ} \mathrm{C}$ during $20 \mathrm{~min}$.

\subsection{Prepare rhizobia culture in liquid nutritional medium (2 days)}

- $\quad$ Take several colonies of R. leguminosarum or S. meliloti from the surface of agar medium with an inoculation loop or sterilized spatula and transfer them to the liquid nutritional medium \# 79 warmed up to $28^{\circ} \mathrm{C}$.

- Grow bacteria at $28^{\circ} \mathrm{C}$ under continuous shaking (100 rpm) in a glass flask with a breathable cover during $48 \mathrm{~h}$ to achieve optical density (OD) of 0.25 at $620 \mathrm{~nm}$.

\subsection{Estimate titer (as optical density, OD) of rhizobial culture (60 min)}


- Pipette $250 \mu \mathrm{L}$ of each rhizobial culture and nutritional medium as control ( $\mathrm{n}=4$ or more) in the wells of a 96-well microtiter plate.

- Determine the titer (OD620) of the bacterial culture spectrophotometrically. If OD620 is $0.250 \pm$ 0.005 , proceed to the next step. If OD620 is higher or lower, centrifuge the suspension (for 10 $\min$ at $4000 \mathrm{~g} / 25^{\circ} \mathrm{C}$ ) and re-suspend the cells in calculated volume of culturing medium. The resulting bacterial culture sediment is diluted with a nutrient solution, the volume of which is calculated by the formula:

$$
\mathrm{V}=\mathrm{dV}_{0} / \mathrm{d}_{\mathrm{a}}
$$

where $V_{0}$ - the initial volume of the bacterial culture solution and the nutrient medium, $V$ - the volume of the nutrient medium solution added after centrifugation, $d$ - is the relative optical density of the initial solution of bacterial culture and the nutrient medium, $d_{a}$ - the target relative optical density of the bacterial culture solution and the nutrient medium at which the concentration of bacterial culture will have desired value. After dilution, a repeated measurement of the optical densityd of the resulting bacterial culture solution is performed. If the measured value of $d$ differs from $d_{a}$, then the procedure described above is repeated until the condition $\left|d-d_{a}\right| \leq \varepsilon_{a}$ is satisfied, where $\varepsilon_{a}$ is \pm 0.005 .

\subsection{Pre-clean of bacterial cells prior to extraction $(60 \mathrm{~min})$}

All works are done in a hood as hexane vapors are toxic.

- Add $0.4 \mathrm{~mL}$ of $n$-hexane to the $2 \mathrm{~mL}$ safe-lock polypropylene tubes, cover the tubes with parafilm and vortex (1000 rpm) for $1 \mathrm{~min}$. Discard $n$-hexane and dry the tubes under air flow.

- Transfer $2 \mathrm{~mL}$ of rhizobial culture to the tubes, and centrifuge for $10 \mathrm{~min}$ at $4000 \mathrm{~g} / 25^{\circ} \mathrm{C}$.

- Discard supernatants, resuspend the pellets in $1 \mathrm{~mL}$ of nutritional medium \# 79, prepared without addition of mannitol- and yeast extract (pH 7.0 - 7.2), vortex (1000 rpm) for $1 \mathrm{~min}$ and centrifuge for $10 \mathrm{~min}$ at $4000 \mathrm{~g}$ and $25^{\circ} \mathrm{C}$. Repeat this procedure two times and discard the supernatant after the last centrifugation.

- Add $1 \mathrm{~mL}$ of $0.9 \%(\mathrm{w} / \mathrm{v}) \mathrm{NaCl}$ to the bacterial pellets, vortex (1000 rpm) for $1 \mathrm{~min}$, centrifuge (10 $\min , 4000 \mathrm{~g}, 25^{\circ} \mathrm{C}$ ) and discard supernatant. Repeat this procedure two times and discard the supernatant after the last centrifugation.

The first pre-cleaning step is required to remove the components of the culture medium and excreted bacterial metabolites, potentially interfering with the analysis of FFAs. The use of the isotonic medium ensures preserving osmotic potential of the rhizobial cells and prevents loss of FFAs due to loss of their integrity.

\subsection{Extract fatty acids from the bacterial cells with $n$-hexane}

Two extraction workflows can be employed to access the FFA fraction of rhizobial cells.

\subsubsection{Extraction from $n$-hexane lyzates ( $15 \mathrm{~min}$ )}

- Supplement the pre-cleaned bacterial pellets with $0.4 \mathrm{~mL}$ of $n$-hexane, cover the tubes with parafilm and vortex (1000 rpm) for $1 \mathrm{~min}$.

- Sonicate the resulted suspension for $2 \mathrm{~min}$ in an ultrasound bath. Add ice to the bath to avoid overheating of the samples.

- Vortex (1000 rpm) the samples for further $2 \mathrm{~min}$, centrifuge (10 min, $4000 \mathrm{~g}, 25^{\circ} \mathrm{C}$ ), and save the supernatant in new $2 \mathrm{~mL}$ safe-lock polypropylene tubes.

\subsubsection{Extraction from aqueous lyzates ( $15 \mathrm{~min}$ )}

- Supplement the pre-cleaned pellets with $0.4 \mathrm{~mL}$ of deionized water and vortex (1000 rpm) for $1 \mathrm{~min}$. 
- Sonicate the resulted suspension during $2 \mathrm{~min}$ in an ultrasound bath. Add ice to the bath to avoid overheating of the samples.

- Add $0.4 \mathrm{~mL}$ of $n$-hexane to the suspensions, cover the tubes with parafilm and vortex (1000 $\mathrm{rpm})$ the samples for further $2 \mathrm{~min}$, centrifuge $\left(10 \mathrm{~min}, 4000 \mathrm{~g}, 25^{\circ} \mathrm{C}\right)$, and save the upper hexane phase in new $2 \mathrm{~mL}$ Safe-Lock polypropylene tubes.

3.6. Apply the samples on a MALDI target (10min per sample, with increase of sample number time requiered for each one is less)

- Apply $0.6 \mu \mathrm{L}$ of the aqueous mixture containing 2,5-dihydroxybenzoic acid (DHB) and barium acetate $(0.25 \mathrm{~g} / \mathrm{L}$ each) on a spot of a polished stainless steel 384-well MALDI target. The mixture forms an aqueous drop within the spot. Make at least three individual applications (technical replicates) at three different spots for each biological replicate.

- Apply $0.6 \mu \mathrm{L}$ of the sample in $n$-hexane on the top central surface of the aqueous drop, containing DHB and barium acetate. Add another $0.6 \mu \mathrm{L}$ portion of the sample after the complete evaporation of hexane.

- Apply $2 \mu \mathrm{L}$ of $90 \%(\mathrm{v} / \mathrm{v})$ aq. acetonitrile after the complete drying of the spot surface (both hexane and aqueous layers need to evaporate completely). Repeat this procedure after reconstitution of the dried monolayer and complete evaporation of the solvent. Formation of symmetric round droplets is desired at this step.

- Using the pipette tip, shift the formed droplet to the edge of the spot and wait for evaporation of the solvent.

- Load the target in the mass spectrometer.

\subsection{Acquire mass spectral data (20 min)}

- Adjust laser energy to $100 \%$. This value will result in better inter-replicate reproducibility.

- Accomplish automatic registration of spectra by the AutoXecute tool in FlexControl software. For this, set acquisition parameters as follows: number of shots accumulated 35000, $\mathrm{m} / \mathrm{z}$ range - 360-550, type of movement - random walk (complete sample), limit diameter of acquisition area $-2000 \mu \mathrm{m}$. Start spectra acquisition.

- Acquire at least three spectra per spot, verify reproducibility of the analysis.

- Open acquired spectra in FlexAnalysis software. Export mass spectral data to .mzXML format to perform following processing of the data.

\subsection{Data processing with MALDI Progenesis software (30 min)}

- Download the necessary data in .mzXML format to Progenesis MALDI.Set the parameter "bin size" to 1.

- Perform pre-processing of the data to remove noise and background artifacts from the spectra. Set the parameters "Noise Filter" and "Background Top Hat filter" to 4 and 60, correspondingly. Press the "Pre-Process all spectra" button and wait until the process is finished. Press Section Complete to move to the next step.

- Perform alignment of the spectra. Set the parameters "Search Area" and "Iterative Cycles" to 5. Press the "Align Spectra" button and wait until the process is finished. Press Section Complete to move to the next step.

- For peak detection choose "Whole Protein" detection method. Set the "Threshold" to 500; set minimum and maximum $\mathrm{m} / \mathrm{z}$ values to 360 and 550 correspondingly. Press the "Detect Peaks" button and wait until the process is finished. Press Section Complete to move to the next step.

- Group technical replicates that represent each biological replicate. To create the sample, select the necessary spectra listed in the right window and press the "Add Selected Spectra to a Sample" button. Fill in the appropriate name of the sample by clicking on the name 
"New Sample 1" in the list on the left. Do the same for the other spectra. Press Section Complete to move to the next step.

- Classify samples into groups. Select the necessary samples from the list in the right window and press the "Add Selected Sample to a Group" button. Click on the name "New Group 1" and fill in the appropriate name of the group. Do the same for the other samples. Press Section Complete to move to the next step.

- Review the results of the analysis. Choose Normalized Peak Height as a statistics measurement for assessing the differences. Choose the type of normalization. Click the tick box on the peaks of interest to put them in the Report. Copy normalization data for peaks of interest to an Excel file for further data analysis by multivariate statistics (these fails can be used for further statistical interpretation - see Section 3.9). The normalization of the data can rely on the total ion current (TIC) and on the peak heights of the signals related to barium monocarboxylates of three typically most abundant in samples fatty acids: palmitic acid $(\mathrm{m} / \mathrm{z}$ 393.14), oleic acid ( $m / z$ 419.16), stearic acid ( $m / z$ 421.17). Press Section Complete to move to the next step.

- Press Section Complete to skip Stats section and move to the next step.

- Create a report from your experimental results. Print the title of your report in the corresponding window and press the "Create Report" button. After that you can view and print your report in the "Report Output" tab. Also, you can save the created report in PDF format by pressing the "Save Report" button.

\subsection{Characterize differences between sample groups by multivariate statistics}

- Rearrange the MS-Excel data exported from Progenesis MALDI at the previous step in the format, compatible with the input requirements of the MetaboAnalyst software tool. For this, create a MS Excel table for each type of normalization. The table needs to contain information on the technical and biological replicates as the first row, sample groups as the second one, and the rest of the rows should contain names of the analytes along with the corresponding normalized peak heights. It is possible to perform analysis using all the replicates separately or using only average peak heights within each technical replicate. For the details of the table arrangement see Supplementary information 1.

- Upload the resulted tables individually to the MetaboAnalyst on-line tool (the option is available in the "Statistical analysis" module). Select the type of data scaling/normalization to access the desired group homogeneity. The following options can be considered: pareto scaling, range scaling, generalized logarithm transformation.

- Accomplish the principal component analysis (PCA) and hierarchical clustering, build corresponding graphs - scores plots and heat maps. Review the results and create a report.

\section{Results and discussion}

To illustrate the potential of our protocol, we performed a comparative study of the FFA metabolomes of two close rhizobial species - Rhizobium leguminosarum bv. viciae RCAM1026 and Sinorhizobium meliloti RCAM1021. For this, the whole workflow was applied to the cultures of these two organisms ( $n=4$, MALDI-TOF-MS analyses were done in three sample application and three spectrum acquisition replicates).

\subsection{Identification of FFAs in rhizobial extracts}

The analyses revealed in total nine fatty acid signals, which could be annotated in the both species by their exact $\mathrm{m} / \mathrm{z}$ values with mass accuracy better than $10 \mathrm{ppm}$ (Table 1), i.e. within the specifications of the instrument. Thereby, the quality and absolute intensities of the acquired spectra (Figure 3) were comparable with those, observed earlier with collapsed solid monolayers of saturated fatty acids [21] and real extracts, obtained from different biological objects, including root nodules of pea (Pisum sativum L.) [25]. It is important to note, however, that lysis under aqueous conditions (Figure 3A and B) yielded approximately doubled spectral intensities and better signal to 
noise ratios in comparison to those obtained after hexane lysis (Figure 3C and D). Most likely, it can be explained with higher efficiency of lipid extraction with $n$-hexane that might compromise crystallization and energy transfer from DHB to the analytes. Comparison of the spectra, acquired from different species under the same conditions (Figure $3 \mathbf{A}, \mathbf{C}$ and $\mathbf{B}, \mathbf{D}$ ), revealed identical qualitative signal patterns, i.e. no FFAs, characteristic for only one of the analyzed species were found. For the most of the analytes, the annotation by $\mathrm{m} / \mathrm{z}$ could be confirmed by tandem mass spectrometry in post-source fragmentation mode (Figure 4). The spectra were informative and allowed localization of double bond in the FA structure, as was described in our previous work [25]. Verification of the observed signals with the spectrum of corresponding authentic standards (as exemplified for linoleic acid in Figure 4) clearly indicated reliability of the MS/MS-based annotation of FFAs in the samples, prepared by means of the Langmuir technology, that is obviously, represents a principal advantage of the method.

Table 1.Annotation of FFAs in $n$-hexane extracts of aqueous and hexane lyzates of Rhizobium leguminosarumbv. viciaeRCAM1026 and SinorhizobiummelilotiRCAM1021cells.

\begin{tabular}{|c|c|c|c|c|c|c|c|c|c|}
\hline \multirow[b]{2}{*}{$\#$} & \multirow{2}{*}{$\begin{array}{c}m / z \\
{[\mathrm{M}-\mathrm{H}+\mathrm{Ba}]^{+}}\end{array}$} & \multirow{2}{*}{$\begin{array}{l}\text { Elemental } \\
\text { composition }\end{array}$} & \multicolumn{5}{|c|}{ Annotationofanalytes } & \multicolumn{2}{|c|}{ Samples } \\
\hline & & & $\begin{array}{c}\text { Annotation } \\
\text { (label) }\end{array}$ & $\begin{array}{l}\text { Error } \\
\text { (ppm) }\end{array}$ & $\begin{array}{l}\text { Isotopic } \\
\text { patterns }\end{array}$ & $\mathbf{M S}^{2}$ & $\begin{array}{c}\text { Tentative } \\
\text { identification }\end{array}$ & $\mathbf{R L}$ & SM \\
\hline 1 & 363.094 & $\mathrm{C}_{14} \mathrm{H}_{25} \mathrm{O}_{2} \mathrm{Ba}^{+}$ & C14:1 & 8.2 & + & - & Myristoleicacid & + & + \\
\hline 2 & 365.108 & $\mathrm{C}_{14} \mathrm{H}_{27} \mathrm{O}_{2} \mathrm{Ba}^{+}$ & $\mathrm{C} 14: 0$ & 5.5 & + & + & Myristicacid & + & + \\
\hline 3 & 377.107 & $\mathrm{C}_{15} \mathrm{H}_{27} \mathrm{O}_{2} \mathrm{Ba}^{+}$ & $\mathrm{C} 15: 1$ & 2.7 & + & - & Pentadecenoicacid & + & + \\
\hline 4 & 379.120 & $\mathrm{C}_{15} \mathrm{H}_{29} \mathrm{O}_{2} \mathrm{Ba}^{+}$ & $\mathrm{C} 15: 0$ & -5.3 & + & + & Pentadecanoicacid & + & + \\
\hline 5 & 391.124 & $\mathrm{C}_{16} \mathrm{H}_{29} \mathrm{O}_{2} \mathrm{Ba}^{+}$ & C16:1 & 5.1 & + & + & Hexadecenoicacid & + & + \\
\hline 6 & 393.137 & $\mathrm{C}_{16} \mathrm{H}_{31} \mathrm{O}_{2} \mathrm{Ba}^{+}$ & C16:0 & -2.6 & + & + & Palmiticacid & + & + \\
\hline 7 & 417.142 & $\mathrm{C}_{18} \mathrm{H}_{31} \mathrm{O}_{2} \mathrm{Ba}^{+}$ & C18:2 & 9.6 & + & + & Linoleicacid & + & + \\
\hline 8 & 419.156 & $\mathrm{C}_{18} \mathrm{H}_{33} \mathrm{O}_{2} \mathrm{Ba}^{+}$ & C18:1 & 7.2 & + & + & Oleicacid & + & + \\
\hline 9 & 421.169 & $\mathrm{C}_{18} \mathrm{H}_{35} \mathrm{O}_{2} \mathrm{Ba}^{+}$ & C18:0 & 2.4 & + & + & Stearicacid & + & + \\
\hline
\end{tabular}

RL, Rhizobium leguminosarum bv. viciae RCAM1026; SM, Sinorhizobiummeliloti RCAM1021

Among the identified FFAs only two structural classes, earlier reported in rhizobia, namely saturated and unsaturated FAs, were detected. No hydroxylated and cyclic acids were detected. However, in general, the observed patterns of FFAs were in agreement with the data obtained with GC-MS. Thus, Theberge et al also identified 16:0, 18:0 and 18:1 acids, although did not report 16:1 and 18:2 acids, described here [28]. A similar pattern was reported by Panday et al in Rhizobium pusense [29], and, in general, confirmed here. It is important to note, that both authors report 19:0 acids (either aliphatic or cyclic), although these analytes could not be detected here. In a specialized Rhizobium species R. selenireduscens, Hunter et al reported 3-hydroxystearic acid [30], which also was not found here. In the cells of R. pseudoryzae, Zhang et al reported 18:1 and 19:0 cyclic acid, as well as methylated and hydroxylated species [31]. Based on the results, obtained here, 18:1 cyclic acids could be potentially present in the cells, analyzed here. Indeed, they are isomeric to 18:2 acids detected here. However, as no separation was used, resolution of isomers is impossible. 
One of the reasons for the absence of methylated and hydroxylated FFAs in the list of annotated here species, can be its low abundance, especially in a FFA form [7]. Indeed, as such acids are, for example, impact on the structure of NOD factors [32], which are low abundant molecules. Thus, to access these acids, NOD factors need to be isolated and hydrolyzed first [10].
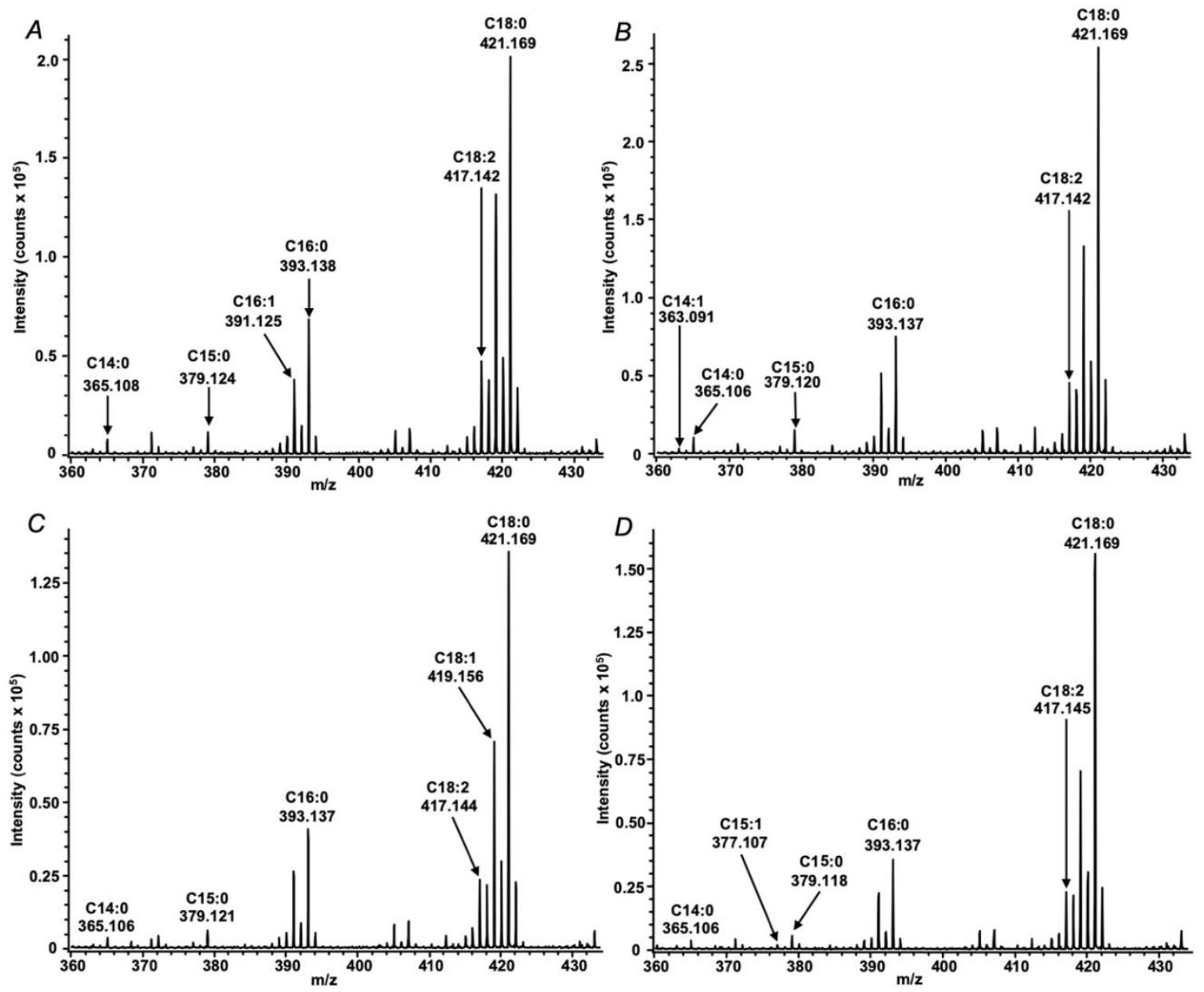

Figure 3.MALDI-TOF mass spectra of extracts obtained by from aqueous (A,B) and $n$-hexane (C,D) lysates of Rhizobium leguminosarum bv. viciae RCAM1026 (A,C) and Sinorhizobiummeliloti RCAM1021 (B,D) cells.

4.2. Relative quantification of FFAs in the R. leguminosarum bv. viciae RCAM1026 and S. meliloti RCAM1021 strains

The homogeneity of the groups and the differences between two species were assessed by the methods of multivariate statistics. The principal question, to be answered at this step was the type of intensity normalization, applied to the acquired raw data. In general, signal intensity depends on the amount of corresponding monocarboxylate at the specific point of a laser shout. In turn, the amount of the ionizable salt is closely related to the conformation of monolayer - if it is straight or folded. As the monolayers, obtained by the Langmuir technology, contain multiple types of FA anions, normalization to its major components might give access to an appropriate correction factor for the variation of the monolayer conformation between spots on the MALDI target. Therefore, based on our previous experience [25], we used the signals at $m / z 393,419$ and 421 , corresponding to palmitic, oleic and stearic acids, as well as the total ion current (TIC) for signal intensity normalization. In 
parallel, we compared two extraction protocols in respect of intra-group dispersion and confidence of the obtained results.

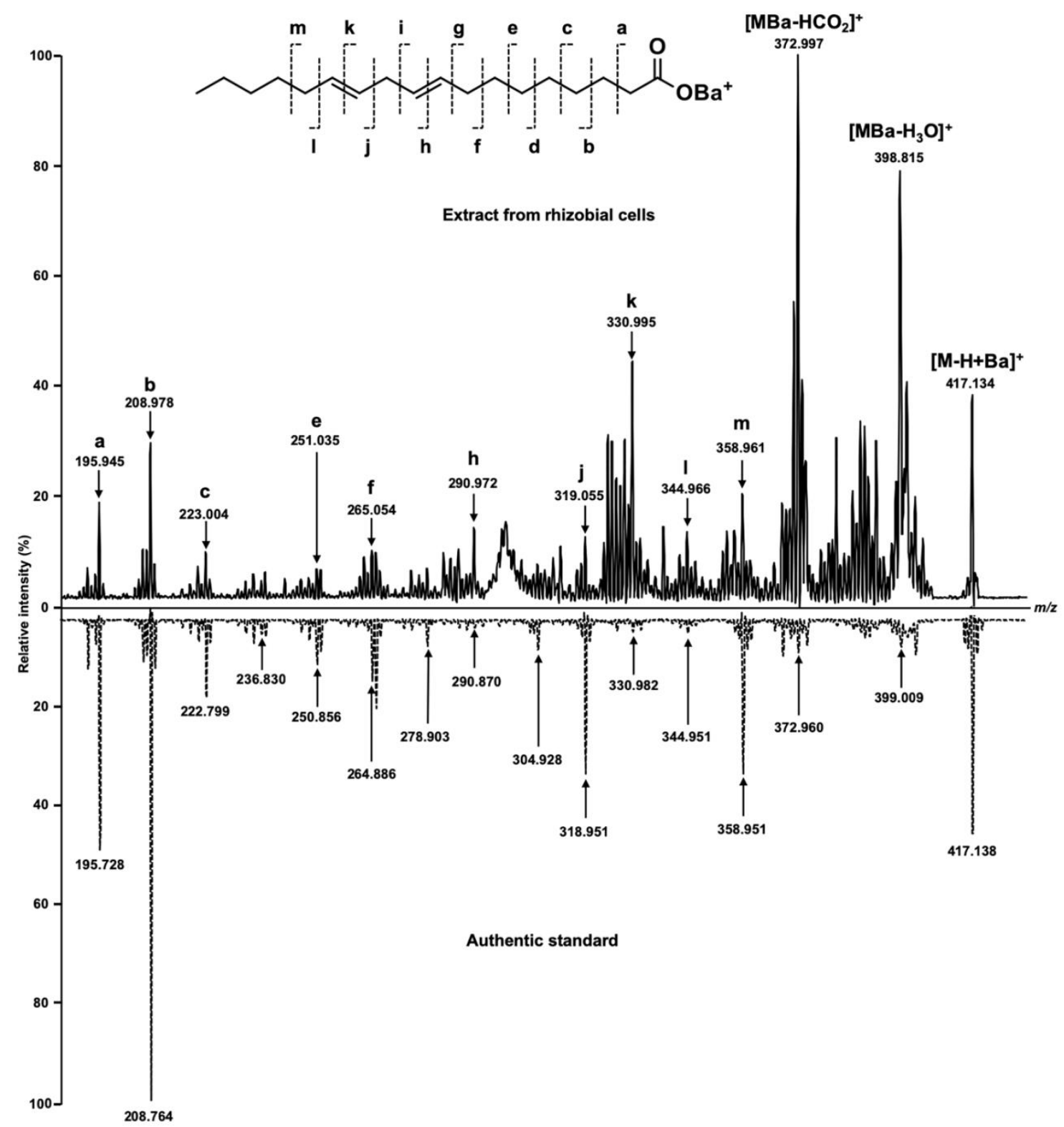

Figure 4. MS/MS spectra of $m / z$ 417.14, corresponding to [M-H+Ba] $]^{+}$ion of linoleic acid. The authentic standard of linoleic acid was dissolved in $n$-hexane and applied on MALDI target in the same way as the extract from rhizobial cells

This comparison revealed the $m / z 421$ as the best-suited normalization signal (Figure 5). Surprisingly, despite lysis with hexane yielded lower signal intensities (Figure 3), it resulted in better separation of two rhizobial species by principal component analysis (PCA, Figure 5A,C) and less dispersion between biological replicates, as can be illustrated by corresponding heat maps (Figure 5B,D). Hierarchical clustering of all individual spectra, including all technical replicates, clearly indicated lysis with hexane as a preferable method in the context of precision and reproducibility (Figure 6). The other normalization strategies (summarized in the Supplementary information 1) gave worth separation between the compared groups. In agreement with this observation, normalization for the $\mathrm{m} / \mathrm{z} 421$ yielded in statistically significant differences in content of FFAs, detected in R. leguminosarum and S. meliloti lyzates. Thereby, six and five differentially 
abundant FFAs were observed when lysis was done with hexane (Supplementary information 2) and water (Supplementary information 3), respectively.
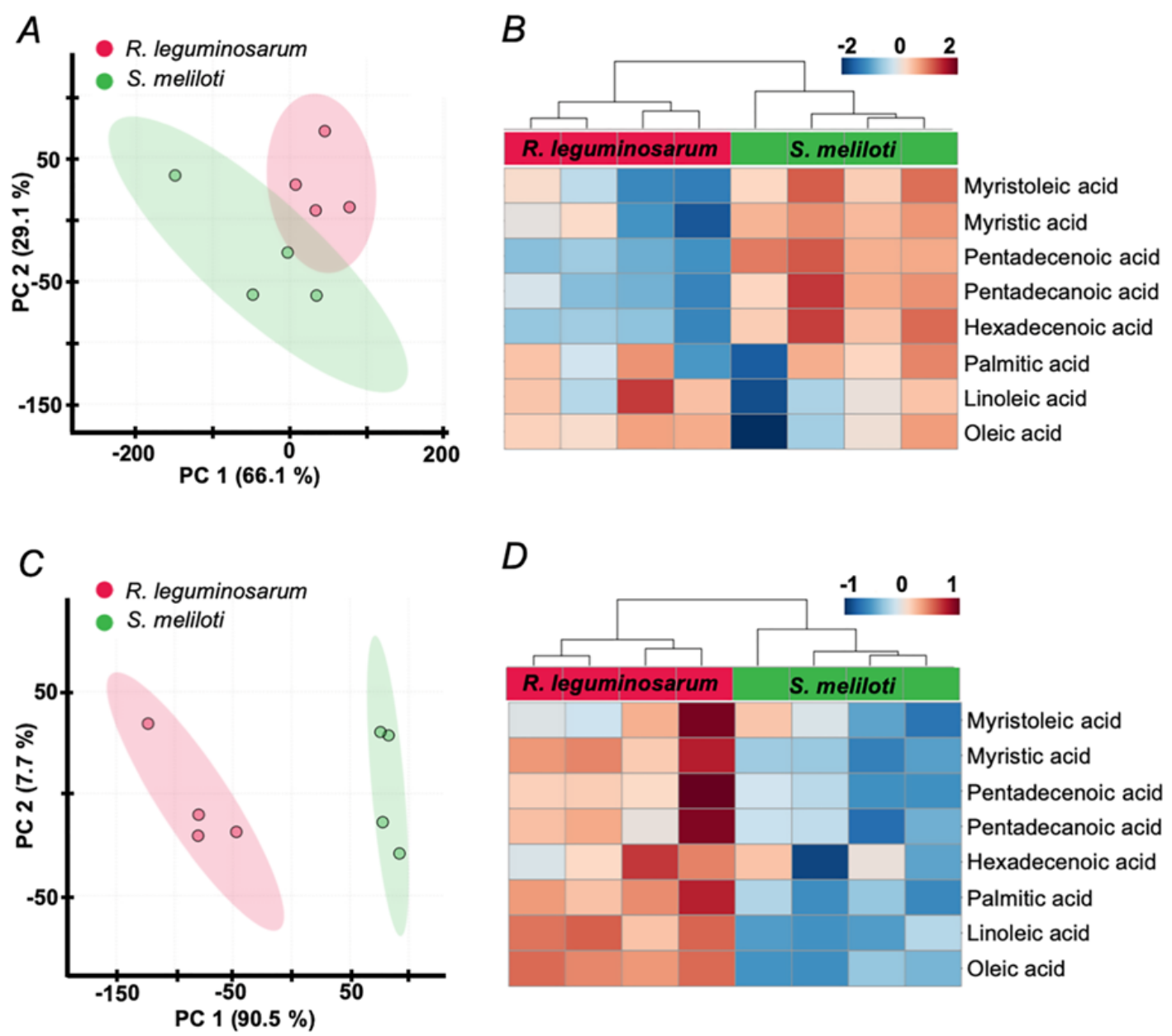

Figure 5. Principal component analysis (PCA) score plots (A and C), built for the first two principal components and heat maps (B and D) for the patterns of FFAs content alterations across R. leguminosarum and S. meliloti samples, obtained by the extraction from aqueous lyzates (A and B) and n-hexane lyzates (C and D). The data were processed in the Progenesis MALDI software with data normalization on the intensities (peak heights) of the signal at $\mathrm{m} / \mathrm{z} 421.17$. The data matrix was further analyzed by the MetaboAnalyst 4.0 on-line software tools. 

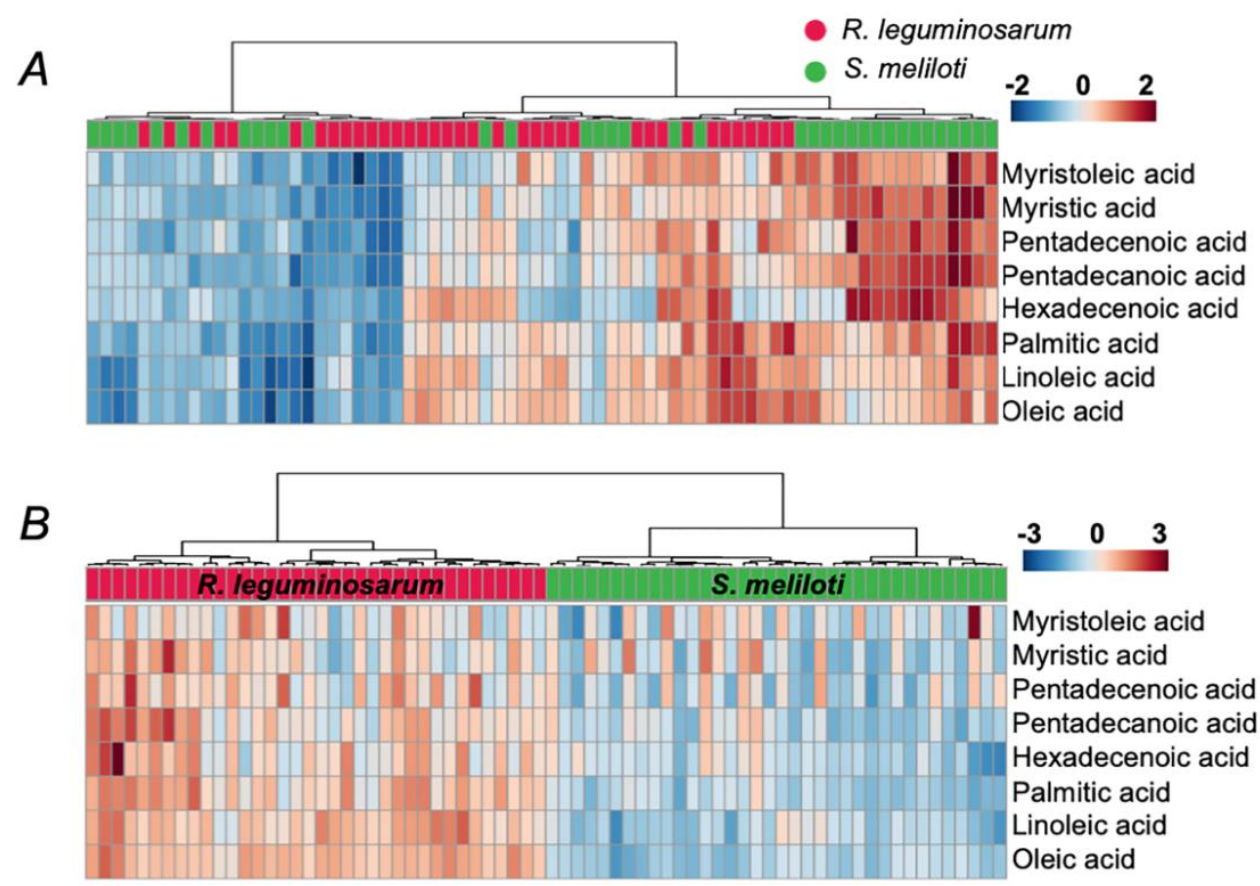

Figure 6. The heat maps for the patterns of FFAs content alterations across all spectra (biological, spotting and acquisition replicates), acquired for R. leguminosarum and S. meliloti samples, obtained by the extraction from aqueous lyzates (A) and n-hexane lyzates (B). The data were processed in the Progenesis MALDI software with data normalization on the intensities (peak heights) of the signal at $\mathrm{m} / \mathrm{z} 421.17$. The data matrix was further analyzed by the MetaboAnalyst 4.0 on-line software tool.

\section{Conclusions}

The presented here protocol represents an essential advantage in analysis of free fatty acids. It is designed as a tool for sensitive, precise and high throughput screening of multiple samples, bacterial species, lines, mutants, ecotypes or specific ecological responses. Similarly to the conventional GC-MS-based approach, our protocol is featured with high sensitivity, precision and accuracy, which is mostly underlined by high regularity of monocarboxylate monolayers, formed directly at the MALDI target. However, application of Langmuir technology in combination with MALDI-TOF-MS allows large batch sizes, which are, basically, restricted only with the capacity of used MALDI targets that typically means 384 analyses including calibration samples. Such a high throughput is hardly accessible by GC-MS, which is, obviously less applicable to large-scale screening experiments. One needs to keep in mind, however, that the absence of chromatographic separation and, hence, inability to separate isomeric acids is the principal intrinsic bottle neck of MALDI-TOF-MS. Thus, complementation of our approach with other methods, giving access for isomer composition of the samples and selected on the basis of preliminary screening, is advantageous.

Supplementary Materials:Supplementary materials can be found at www.mdpi.com/xxx/s1.

Author Contributions: AG impacted on the development and filming the protocol, did sample preparation, accomplished MALDI-TOF-MS analysis and processing, contributed in writing the first draft; JS and AK impacted on the development and filming the protocol, accomplished rhizobia culturing, extraction of free fatty acids and contributed in writing the first draft; KB performed statistical interpretation of the data, contributed in writing of the first draft; AT and IA worked on video design and sounding; LL supervised the works with bacteria; VAZ and IAT contributed in writing final draft and critical reading; CB contributed with methodology and impacted on the final manuscript draft; EP contributed in the methodology, protocol and video conceptualization, supervised sample preparation and MS experiments; AF conceptualized the manuscript, supervised the whole work and contributed in writing of the final draft. 
Funding: The authors acknowledge the funding from the Russian Foundation of Basic Research (RFBR, grant \# 18-016-190A) for JS, LL, AT and AF, from Deutsche Forschungsgemeinschaft (DFG, grant \# FR3417-2/3) for AF, from Russian Science Foundation (RSF, grant \# 17-76-30016) for VAZ and IAT and from the government of Russian Federation (grant \# 0074-2014-0012 State registration number: AAAA-A16-116041310010-6) for AG and EP.

Acknowledgments: The Research Resource Center for Molecular and Cell Technologies of St. Petersburg State University is acknowledged for technical support. Authors thank Polina Dubakova (Institute of Toxicology, Federal Medical-Biological Agency of Russia), Dmitri Efimov (Aviarobots LLC) and Alexander Protasov (Research Institute of Influenza, St. Petersburg, Russia) for assistance in filming this protocol.

Conflicts of Interest:The authors declare no conflict of interest.

$\begin{array}{ll}\text { Abbreviations } & \\ \text { DGTS } & \text { diacylglyceryl-N,N,N-trimethylhomoserine } \\ \text { DHB } & \text { 2,5-dihydroxybenzoic acid } \\ \text { ESI-MS } & \text { electrospray ionization mass spectrometry } \\ \text { FAs } & \text { fatty acids } \\ \text { FFAs } & \text { gree fatty acids } \\ \text { GC-MS } & \text { hydrophilic interaction liquid chromatography } \\ \text { HILIC } & \text { matrix-assisted laser desorption/ionization-time of flight mass spectrometry } \\ \text { MALDI-TOF-MS } & \text { multiple reaction monitoring mode } \\ \text { MRM } & \text { tandem mass spectrometry } \\ \text { MS/MS } & \text { nodulation } \\ \text { Nod } & \text { optical density } \\ \text { OD } & \text { principal component analysis } \\ \text { PCA } & \text { reversed phase high-performance liquid chromatography } \\ \text { RP-HPLC } & \text { total ion current }\end{array}$

\section{References}

1. Fulco, A.J. Metabolic alterations of fatty acids. Annu Rev Biochem.1974,43,215-241; DOI:10.1146/annurev.bi.43.070174.001243.

2. Ratnayake, W.M; Galli, C. Fat and fatty acid terminology, methods of analysis and fat digestion and metabolism: a background review paper.Ann NutrMetab. 2009,55,8-43; DOI:10.1159/000228994.

3. Balcke, G.U.;Handrick, V.;Bergau, N.;Fichtner, M.; Henning, A.;Stellmach, H.;Tissier, A.;Hause, B.;Frolov, A. An UPLC-MS/MS method for highly sensitive high-throughput analysis of phytohormones in plant tissues.Plant Methods. 2012, 8, 47; DOI:10.1186/1746-4811-8-47.

4. Bosviel, R.; Joumard-Cubizolles, L.; Chinetti-Gbaguidi, G.; Bayle, D.; Copin, C.; Hennuyer, N.; Duplan, I.; Staels, B.; Zanoni, G.; Porta, A.; Balas, L.; Galano, JM.; Oger, C.; Mazur, A.; Durand, T.; Gladine, C. DHA-derived oxylipins, neuroprostanes and protectins, differentially and dose-dependently modulate the inflammatory response in human macrophages: Putative mechanisms through PPAR activation. Free Radical Bio Med.2017,103, 146-154; DOI:10.1016/j.freeradbiomed.2016.12.018.

5. Mhamdi, R.; Nouairi, I.; ben Hammouda, T.; Mhamdi, R.; Mhadhbi, H. Growth capacity and biochemical mechanisms involved in rhizobia tolerance to salinity and water deficit. J Basic Microbiol. 2015, 55, 451-461; DOI:10.1002/jobm.201400451.

6. Goettel, M.; Niessner, R.; Pluym, N.; Scherer, G.; Scherer, M.A fully validated GC-TOF-MS method for the quantification of fatty acids revealed alterations in the metabolic profile of fatty acids after smoking cessation. J. Chromatogr. B: Anal. Technol. Biomed. Life Sci.2017, 1041-1042, 141-150; DOI:10.1016/j.jchromb.2016.12.035.

7. O'Leary, W.M. The fatty acids of bacteria. Bacteriol Rev. 1962, 26, 421-447.

8. López-Lara, I.M.; Geiger, O. Bacterial lipid diversity. BiochimBiophys Acta Mol Cell Biol Lipids. 2017, 1862, 1287-1299; DOI:10.1016/j.bbalip.2016.10.007.

9. Matamoros, M.A.; Kim, A.;Peñuelas, M.;Ihling, C.;Griesser, E.;Hoffmann, R.;Fedorova, M.;Frolov, A.;Becana, M.Protein Carbonylation and Glycation in Legume Nodules.Plant Physiol. 2018,177, 1510-1528. DOI:10.1104/pp.18.00533. 
10. Demont, N.; Ardourel, M.; Maillet, F.; Promé, D.; Ferro, M.; Promé, JC.; Dénarié, J. The Rhizobium meliloti regulatory nodD3 and syrM genes control the synthesis of a particular class of nodulation factors $\mathrm{N}$-acylated by ( $\omega$-1)-hydroxylated fatty acids. Embo J. 1994, 13, 2139-2149.

11. Zarifikhosroshahi, M.; Murathan, Z.T.; Kafkas, E.; Okatan, V. Variation in volatile and fatty acid contents among Viburnum opulusL. Fruits growing different locations. Sci Hortic.2020, 264, 109160; DOI:10.1016/j.scienta.2019.109160.

12. Bianchi, F.; Dall'Asta, M.; Del Rio, D.; Mangia, A.; Musci, M.; Scazzina, F. Development of a headspace solid-phase microextraction gas chromatography-mass spectrometric method for the determination of short-chain fatty acids from intestinal fermentation. Food Chem.2011, 129, 200-205. DOI: /10.1016/j.foodchem.2011.04.022.

13. Dernekbaşı, S.;Oksüz, A.;C,elik, M.Y.; Karayücel, I.; Karayücel, S. The Fatty Acid Composition of Cultured Mussels (Mytilusgalloprovincialis Lamarck 1819) in Offshore Longline System in the Black Sea. J Aquac Mar Biol.2015, 2, 00049; DOI: 10.15406/jamb.2015.02.00049.

14. Sheet, S.; Yesupatham, S.; Ghosh, K.; Choi, MS.; Shim, KS.; Lee, YS. Modulatory effect of low-shear modeled microgravity on stress resistance, membrane lipid composition, virulence, and relevant gene expression in the food-borne pathogen Listeria monocytogenes. Enzyme Microb Technol. 2020, 133, 109440; DOI:10.1016/j.enzmictec.2019.109440.

15. Krylov, A. I.; Khlebnikova, N. S.; Poluyaktova, S. K. Gas chromatographic determination of free fatty acids of blood using extractive alkylation. J. Anal. Chem.1991, 46, 2428-2435 [in Russian].

16. Christie, W. W. Advances in Lipid Methodology - Two; Oily Press Ltd.: Dundee, Scotland, 1993; pp. 69-111.

17. Gallart-Ayala, H.; Konz, I.; Mehl, F.; Teav, T.; Oikonomidi, A.; Peyratout, G.; van der Velpen, V.; Popp, J.; Ivanisevic, J. A global HILIC-MS approach to measure polar human cerebrospinal fluid metabolome: Exploring gender-associated variation in a cohort of elderly cognitively healthy subjects. Anal Chim Acta. 2018, 1037, 327-337; DOI:10.1016/j.aca.2018.04.002.

18. Zhang, T.; Chen, S.; Syed, I.; Ståhlman, M.; Kolar, M.J.; Homan, E.A.; Chu, Q.; Smith, U.; Borén, J.; Kahn, B.B.; Saghatelian, A. A LC-MS-based workflow for measurement of branched fatty acid esters of hydroxy fatty acids. Nat Protoc. 2016, 11,747-763; DOI:10.1038/nprot.2016.040.

19. Serafim, V.; Tiugan, D.-A.; Andreescu, N.; Mihailescu, A.; Paul, C.; Velea, I.; Puiu, M.; Niculescu, M.D. Development and Validation of a LC-MS/MS-Based Assay for Quantification of Free and Total Omega 3 and 6 Fatty Acids from Human Plasma. Molecules. 2019, 24, 360; DOI:10.3390/molecules24020360.

20. Yi, L.; He, J.; Liang, Y.; Yuan, D.; Gao, H.; Zhou, H. Simultaneously quantitative measurement of comprehensive profiles of esterified and non-esterified fatty acid in plasma of type 2 diabetic patients. Chem. Phys. Lipids. 2007, 150, 204-216; DOI:10.1016/j.chemphyslip.2007.08.002.

21. Podolskaya, E.P.; Serebryakova, M.V.; Krasnov, K.A.; Grachev, S.A.; Gzgzyan, A.M.; Sukhodolov, N.G. Application of Langmuir-Blodgett technology for the analysis of saturated fatty acids using the MALDI-TOF mass spectrometry. Mendeleev Commun. 2018, 28, 337-339; DOI:10.1016/j.mencom.2018.05.037ю

22. Gladilovich, V.; Greifenhagen, U.; Sukhodolov, N.; Selyutin, A.; Singer, D.; Thieme, D.; Majovsky, P.; Shirkin, A.; Hoehenwarter, W.; Bonitenko, E.; Podolskaya, E.; Frolov, A. Immobilized metal affinity chromatography on collapsed Langmuir-Blodgett iron(III) stearate films and iron(III) oxide nanoparticles for bottom-up phosphoproteomics. J. Chromatogr. A.2016, 1443, 181-190;DOI:10.1016/j.chroma.2016.03.044.

23. Babakov, V.N.; Shreiner, E.V.; Keltsieva, O.A.; Dubrovskii, Y.A.; Shilovskikh, V.V.; Zorin, I.M.; Sukhodolov, N.G.; Zenkevich, I.G.; Podolskaya, E.P.; Selyutin, A. Lanthanum stearate monolayers as a metal-affinity sorbent for the selective sorption of soman adducts to human serum albumin. Talanta, 2019, 195, 728-731; DOI:10.1016/j.talanta.2018.11.103.

24. Shreyner, E.V.; Alexandrova, M.L.; Sukhodolov, N.G.; Selyutin, A.A.; Podolskaya, E.P. Extraction of the insecticide dieldrin from water and biological samples by metal affinity chromatography. Mendeleev Commun., 2017, 27, 304-306; DOI:10.1016/j.mencom.2017.05.030.

25. Podolskaya, E.P.; Gladchuk, A.S.; Keltsieva, O.A.; Dubakova, P.S.; Silyavka, E.S.; Lukasheva, E.; Zhukov, V., Lapina, N.; Makhmadalieva, M.R.; Gzgzyan, A.M.; Sukhodolov, N.G.; Krasnov, K.A.; Selyutin A.A.; Frolov, A. Thin film chemical deposition techniques as a tool for fingerprinting of free fatty acids by MALDI-TOF-MS. Anal Chem. 2019, 91, 1636-1643; DOI:10.1021/acs.analchem.8b05296.

26. Matharu, Z.; Bandodkar, A.J.; Gupta, V.; Malhotra, B.D. Fundamentals and application of ordered molecular assemblies to affinity biosensing. ChemSoc Rev. 2012,41, 1363-1402; DOI:10.1039/c1cs15145b. 
27. Stickland, F.G.W. The formation of monomolecular layers by spreading a copper stearate solution. J.ColloidInterfaceSci. 1972, 40, 142-152; DOI:10.1016/0021-9797(72)90003-3.

28. Theberge, MC.; Prevost, D.; Chalifour, FP. The effect of different temperatures on the fatty acid composition of Rhizobium leguminosarumbv. viciae in the faba bean symbiosis. The New Phytologist. 1996, 134, 657-664; DOI:10.1111/j.1469-8137.1996.tb04931.x.

29. Panday, D.; Schumann, P.; Das, SK. Rhizobium pusense sp. nov., isolated from the rhizosphere of chickpea (Cicer arietinum L.).Int J SystEvolMicrobiol.2011, 61, 2632-2639; DOI:10.1099/ijs.0.028407-0.

30. Hunter, W.J.; Kuykendall, L.D.; Manter, D.K. Rhizobiumselenireducenssp. nov.: a selenite-reducingalpha-Proteobacteriaisolatedfrom a bioreactor. CurrMicrobiol.2007, 55, 455-60; DOI:10.1007/s00284-007-9020-9.

31. Zhang, X.; Sun, L.; Ma, X.; Sui, X.H; Jiang, R. Rhizobiumpseudoryzaesp. nov., isolatedfromtherhizosphereofrice.Int J SystEvolMicrobiol. 2011, 61, 2425-2429; DOI:10.1099/ijs.0.026146-0.

32. Becker, A.; Fraysse, N.; Sharypova, L. Recent advances in studies of structure and simbiosis-related function of Rhizobial K-antigens and lipopolysaccharides. MPMI. 2005, 18, 899-905; DOI: 10.1094/MPMI -18-0899. 